\title{
Banked human milk treatment and immunoactive factors content. Comparison with high pressure processing
}

\author{
A. Franch ${ }^{1,2}$, C. Audí $^{1,2}$, C. Ramírez-Santana ${ }^{1,2}$, M. Permanyer ${ }^{3}$, F. J. Pérez-Cano ${ }^{1}$, \\ C. Moltó-Puigmarti ${ }^{2,4}$, M. C. López-Sabater ${ }^{2,4}$ and C. Castellote ${ }^{1,2}$ \\ ${ }^{1}$ Department of Physiology, Faculty of Pharmacy, University of Barcelona, Spain, ${ }^{2}$ CIBER in Epidemiology and Public \\ Health (CIBERESP), Spain, ${ }^{3}$ Food New Technologies and Processes Center (CENTA-IRTA), Monells (Girona), Spain and \\ ${ }^{4}$ Department of Nutrition and Food Science, Faculty of Pharmacy, University of Barcelona, Spain
}

Breast-feeding is an unequalled way of providing ideal food for the healthy growth and development of infants. As a global public health recommendation, infants should be exclusively breastfed for the first 6 months of life to achieve optimal growth, development and health. For those situations where infants cannot be breastfed, stored breast milk from a human-milk bank constitutes an excellent alternative. The aim of this work was to evaluate the effect of the Human Milk Banking procedure ${ }^{(1)}$ on the content of IgA, EGF, TGF- $\beta 1$, TGF- $\beta 2$, IL-6, IL-8, IL-10, IL-13, TNF- $\alpha$ and TNF-RI. Moreover, a comparison with high hydrostatic pressure (HHP) milk processing has been performed.

Mature human milk samples $(n=10)$ were obtained from healthy volunteer donors of ALBA Barcelona Association. All women delivered at term between the 38th and 40th week of gestation. Milk samples were collected from both breasts and each milk sample was initially divided in three groups: raw-unprocessed (RM), pasteurized (PT) and HHP processed (HHP) human milk. Samples were subjected to heat $\left(62.5^{\circ} \mathrm{C}\right.$ for $\left.30 \mathrm{~min}\right)$ in PT group or to 400,500 and $600 \mathrm{MPa}\left(5 \mathrm{~min}\right.$ at $10-12^{\circ} \mathrm{C}$; Wave $6000 / 120$, NC Hyperbaric) highpressure procedures in HHP group. Milk whey was obtained by centrifugation at $800 \mathrm{~g}, 10 \mathrm{~min} 4^{\circ} \mathrm{C}$. IgA, EGF, TGF- $\beta 1$ and TGF- $\beta 2$ were determined by ELISA assays, and IL-6, IL-8, IL-10, IL-13, TNF- $\alpha$ and TNF-RI were quantified by BD ${ }^{\text {TM }}$ Cytometric Bead Array Human Soluble Flex Set Assay (Becton Dickinson, San Diego, CA, USA). Non-parametric test for paired samples were used, specifically Friedman test and Wilcoxon $T$ test. Significant differences were accepted at $P<0.05$.

As expected, IgA content was significantly reduced by thermal treatment $(P<0.05)$, but HHP at $400 \mathrm{MPa}$ maintained IgA milk whey concentration. Immunoactive components such as EGF, TGF- $\beta 1$ and TGF- $\beta 2$ were not modified by either PT or HHP treatment. Pasteurisation induced a significant increase of IL-8 $(P<0.05)$ and a reduction of IL-6, TNF- $\alpha$ and IL-13 $(P<0.05)$. However, the HHP treatment at $400 \mathrm{MPa}$ did not produce the changes above mentioned (Fig. 1).

As HHP milk processing is known to maintain macronutrients, in relation to the immunoactive factors studied in this work, pressure treatment could be applied as an alternative to heating in human milk-banking.

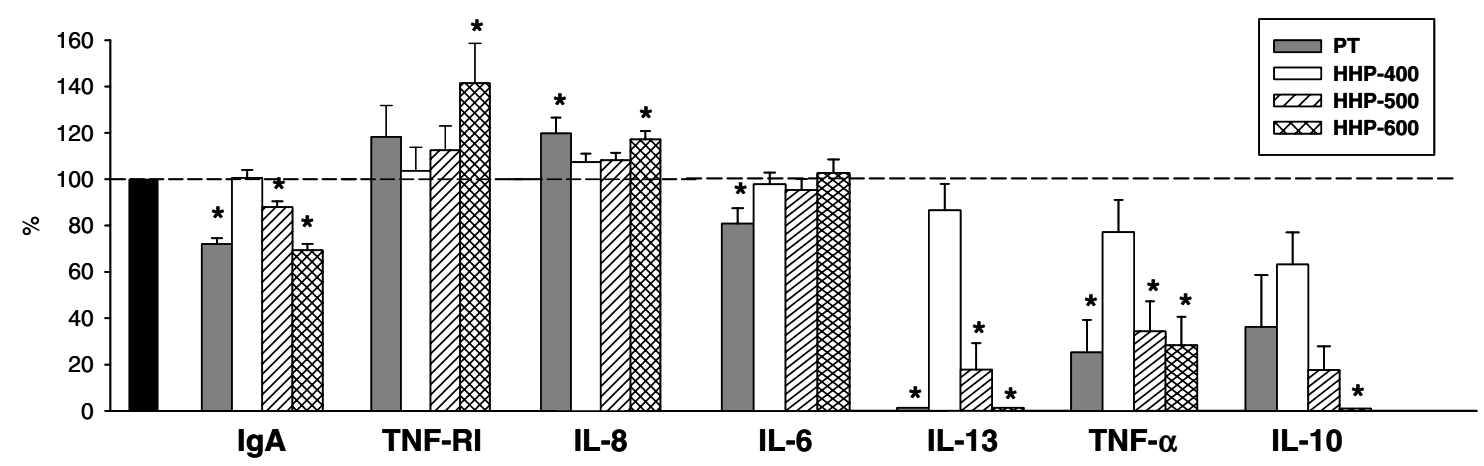

Fig. 1. Retention percentage of immunoactive components of human breast milk after pasteurisation or HHP treatments. Means \pm SE. $* P<0.05 v$. raw-unprocessed milk (black bar).

1. Human Milk Banking Association of North America (HMBANA) (2005) Available at http://www.hmbana.org/ 